\title{
Bericht über die internationale Tagung Grenzen der Sprache Grenzen der Sprachwissenschaft (= Linguistische Treffen in Wrocław VI) am Institut für Germanistik der Universität Wrocław, 14.-16. September 2017
}

Krystian Suchorab

Vom 14. bis zum 16. September 2017 fand am Institut für Germanistik der Universität Wrocław die internationale linguistische Tagung Grenzen der Sprache, Grenzen der Sprachwissenschaft statt. Es war schon das sechste Treffen der Germanisten aus aller Welt im Rahmen des im Jahre 2006 initiierten Zyklus Linguistische Treffen in Wroctaw. Die Tagung wurde vom Lehrstuhl für Deutsche Sprache und Lehrstuhl für Angewandte Linguistik des Instituts für Germanistik der Universität Wrocław veranstaltet.

Die feierliche Eröffnung der Tagung fand am 14. September 2017 im prächtigen Nehring-Saal der philologischen Fakultät statt. Die Teilnehmer wurden Prof. Dr. Iwona Bartoszewicz, der Prorektorin der Universität Wrocław, Prof. Dr. Marcin Cieński, dem Dekan der Philologischen Fakultät, Prof. Dr. Tomasz Małyszek, dem Leiter des Instituts für Germanistik und Dr. habil. Joanna Szczęk, der Leiterin des Lehrstuhls für Angewandte Linguistik begrüßt. In den Grußreden wurde betont, dass der Einladung der Organisatoren wie immer viele Sprachwissenschaftler aus der ganzen Welt, darunter auch Gäste aus drei Kontinenten gefolgt sind. Nach den Grußworten fingen die Plenarvorträge an. Oleksandr M. Bilous (Kropywnyzkyj) sprach zum Thema: Wechselseitigkeit literarischen Kulturgutes: historischer Blick. Danach folgte die Präsentation von Tomasz Jabłecki (Wrocław): Zum Phänomen der Rezeptionsliteratur im 17. Jahrhundert am Beispiel Schlesiens. Im Vortrag wurde betont, dass die Rezeption der Literatur u. a. den Transfer von fremden Ideen, anderen Sprach- und Kulturbereichen und bestimmten Faktoren bedeutet. Der dritte Plenarvortrag wurde von Jan Pacholski (Wrocław) zum Thema: Alle Facetten eines Grenzlandes par excellence - das Riesengebirge im Schriftum des ausgehenden 18. Jahrhunderts gehalten. Im Referat wurden die Facetten des Grenzlandes Riesengebirge und die Rolle des Riesengebirges in den schriftlichen Erwähnungen dargestellt. 
Die Referate an den nächsten Tagen wurden in vier parallele Sektionen eingeteilt. Die erste Sektion war der Sprache im öffentlichen Raum gewidmet. Hier sprach Mariusz Jakosz (Sosnowiec) zum Thema: Ironie als Ausdrucksmittel des Bewertens im Deutschen Online-Diskurs über deutsch-polnische Beziehungen. Er behandelte solche stilistischen Sprachmittel wie Metaphern, Ironie, Vergleiche oder Sprachspiele, die als Indikatoren gelten können. Danach folgte die Präsentation Sprecherwechsel in den deutschen und lettischen politischen Fernsehtalkshows von Jūlija Zālīte (Riga). Anhand der ausgewählten Transkripte der deutschen und lettischen politischen Talkshows versuchte sie zu zeigen, wie die Gesprächsteilnehmer den Sprecherwechsel in einer so komplizierten Gesprächsform realisieren. Michaela Schnick (Dresden) sprach zum Thema: Sprachliche Grenzen in Radikalisierungsprozessen. Im Referat näherte sie sich über die Analyse sprachlicher Feindbildkonstruktionen dem Wechselspiel zwischen Grenzziehungen und Grenzüberschreitungen. Das nächste Referat: Wörter ohne Grenzen. Zur Form und Leistung der Komposita in den Überschriften der Presseartikel am Beispiel der Online-Ausgabe der Wochenzeitung „Die Zeit“ wurde von Anna Dargiewicz (Olsztyn) gehalten. In der Präsentation wurde erläutert, welche Rolle die Komposita in Überschriften spielen. Dann sprach Natalia Sineokaia (Rostow am Don) zum Thema: Weiblicher politischer Diskurs in dem deutschen medialen Raum. Der nächste Beitrag wurde von Hanna Stypa (Bydgoszcz) zum Thema: Zum Computerspielerjargon in der Chat-Kommunikation gehalten. Sie thematisierte die Spezifik der Chat-Kommunikation der Computerspieler und die charakteristischen Merkmale ihres Jargons.

Die Sektion zwei diskutierte zum Rahmenthema: Sprechen und Kommunizieren im Vergleich. Der erste Beitrag in dieser Sektion zum Thema: Zwischen Bedeutungen und Verstehen. Semantische Grenze in der Sprache in glottodidaktischer Perspektive wurde von Adam Szeluga (Gdańsk) gehalten. Im Referat wurde das Problem der semantischen Grenze in der Sprache aus der Sicht der Fremdsprache und der Fremdsprachendidaktik dargestellt. Danach folgte das Referat von Agnieszka Pawłowska-Balcerska (Poznań): Internationale Lehrkooperationen am Beispiel eines polnisch-deutschen E-Mail-Tandemprojekts mit fortgeschrittenen Deutschlernenden und angehenden DaF-Lehrenden - im Spannungsfeld von Möglichkeiten und Grenzen. Nach diesem Referat sprach Junko Nakagawa (Tokyo) zum Thema: Zur Reformierung der Ausspracheschulung für DaF an den Hochschulen. In diesem Vortrag ging sie auf eine Diskussion zur Reformierung der Ausspracheschulung für DaF an den Hochschulen. Das nächste Referat wurde von Ofeliya Mustafayeva (Wien) zum Thema: Kontrastiver Vergleich von Tierphraseologismen aus der Sicht der Sprach- und Übersetzungswissenschaft. Dargestellt anhand deutschsprachiger Literatur und deren aserbaidschanischer Übersetzungen gehalten. Hier wurde berührt, dass man beim Übersetzen von verschiedenen Texten oft auch verschiedene Schwierigkeiten stößt. Daniela Pelka (Opole) stellte ihren Beitrag zum Thema Treffen sich ein Pole und ein Deutscher: Zu den Schwierigkeiten bei der Übersetzung von Witzen dar.

Die dritte Sektion hatte ein folgendes Rahmenthema: Konvention und Innovation im Interagieren linguistisch erfassen. Das erste Referat wurde von Jolanta MazurkiewiczSokołowska (Szczecin) zum Thema: Introspektion als Untersuchungsmethode in der kognitionsorientierten Sprachforschung gehalten. Dieser Beitrag hatte zum Ziel, vor dem Hintergrund der methodologischen Schwierigkeiten, die Vor- und Nachteile der Introspekti- 
on zu diskutieren. Die nächste Präsentation von Jany Berit (Colorado) trug den Titel: Interimssprache und das Bewusstmachen von Sprachfähigkeit und die Autorin präsentierte Ergebnisse eines Projekts, die unter anderem größere Transparenz und selbstgesteuertes Lernen anregen, die den Lernern bereits entwickelte Fähigkeiten der Interimssprache bewusst machen und somit die Lernmotivation erheblich steigern. Danach folgte die Präsentation von Attila Péteri (Budapest) zum Thema: Epistemik im Korpus. Methoden der korpuslinguistischen Erfassung von Sprechereinstellungen. Er präsentierte eine Analysemethode eines thematischen Pressekorpus anhand des sog. „Budapester Korpus“. Der nächste Vortrag wurde von Joanna Pędzisz (Lublin) zum Thema: Tänzer als Diskursgemeinschaft: Dialog über Tanz im Spannungsfeld zwischen Sprache, Körper und Bewegung. Sie stellte eine solche Frage: „Wie kommunizieren Tänzer als Diskursgemeinschaften?“ und versuchte sie zu beantworten. Dann kam Elizveta Kotorova (Zielona Góra) mit ihrem Referat zum Thema: Liegt Pragmatik in Grenzen der Sprachwissenschaft? zu Wort. Sie versuchte die Frage zu beantworten, ob in der Struktur der Sprache eine pragmatische Ebene anerkannt werden kann. Danach folgte ein Beitrag von Piotr Krycki (Zielona Góra) zum Thema: Grenzen der sozialen Systeme - Grenzen der Kommunikation?. Dieser Beitrag sollte u.a. zeigen, wie Textlinguistik und Systemtheorie einander produktiv ergänzen können. Dann wurde das Referat von Piotr Bartelik (Zielona Góra) zum Thema: Zu den Grenzen der grammatischen (Re)Analyse gehalten. Dieser Vortrag setzte sich zum Ziel die Untersuchung der divers bedingten Objektselektion in polnischen periphrastischen mieć-Formen. Danach sprach Anna Hopp (Olsztyn) zum Thema: Vom Aussterben bedrohte Wörter. Der letzte Beitrag in dieser Sektion wurde von Angela Schmidt-Berhnardt (Marburg) und Sylwia Adamczak-Krysztofowicz (Poznań) zum Thema: Sprechängste als mögliche Ursache für Grenzen der Kommunikation am Beispiel multikultureller Begegnungsprojekte an Hochschulen gehalten.

Die Sektion Nummer vier thematisierte Varietäten. Das erste Referat wurde von Linda Yoksulabakan (Mainz) zum Thema: Identitätskonstruktion indigener und nicht-indigener Hetäre in multimodalen, offizielen Ego-Dokumenten': Eine Untersuchung der Sprachgeschichte,von unten' des Spanischen in Oaxaca, Mexiko (XIX. Jhd.) gehalten. Danach folgte ein Vortrag von Jannis Harjus (Innsbruck) zum Thema: Neue methodische Wege der Varietätenlinguistik: sequentielles mixed-emische Methodentriangulation. Dieser Beitrag präsentierte einen neuen methodischen Ansatz aus der Romanistik, sich innerhalb der Varietätenlinguistik deskriptiven Sprachnormen zu nähern. Das nächste Referat wurde von Małgorzata Derecka (Olsztyn) zum Thema: Der deutsche Rap - das Sprachrohr der deutschen Minderheiten oder eine Rechtfertigeung der mangelhaften Sprachkenntnisse gehalten. Hier wurde berührt, dass die besondere Mischung von vielen Sprachen, Dialekten, Umgangssprache, Neubildungen, Abkürzungen ein Code zur Verschiebung der inneren mentalen Grenze zu sein scheint. Der nächste Vortrag wurde von Grażyna Strzelecka (Warszawa) zum Thema: Zur Wirtschaftssprache des ausgehenden 19. Jahrhunderts - Bezeichnungen aus dem Bereich Arbeit und Soziales in ausgewählten Pressezitaten gehalten. Im Beitrag wurden ausgewählte Bezeichnungen aus Pressetexten aus den Jahren 1885-1886 präsentiert und mit Hilfe von Wirtschaftswörterbüchern definiert und beschrieben. Danach sprach Federico Collaoni (Udine) zum Thema: Grenzen der Sprache und Grenzen der Sprachwissenschaft in 
der Ökolinguistik. Das nächste Referat wurde von Monika Schönherr (Zielona Góra) zum Thema: Meinungsbekundungen im akademischen Diskurs gehalten. Der Vortrag befasste sich mit dem akademischen Schreiben polnischer Studierenden im Fach Germanistik als Fremdsprachenphilologie. Der letzte Beitrag in dieser Sektion wurde von Mihály Harsányi (Eger) zum Thema: Untersuchung adjektivischer Bildungen auf „-schwanger“ gehalten. Im Vortrag wurden die Ergebnisse einer synchronischen Untersuchung dieser Bildungen.

Darüber hinaus fand an dem zweiten Tag der Tagung eine Führung durch das Hauptgebäude der Universität Wrocław statt und wurden Plenarvorträge gehalten. Der erste Plenarvortrag wurde von Michail L. Kotin (Zielona Góra) zum Thema: Die Grenze der Erkenntnis in der Linguistik: Ontogenese und Phylogenese dargestellt. Danach folgte der zweite Plenarvortrag von Tomáš Hoskovec (Prag) zum Thema: ... und wenn die Sprache einmal genügend abgegrenzt wird?. In diesem Vortrag wollte der Redner, die Abgrenzung der Sprache schrittweise erklären, und auf das ethische sowie ästhetische Ausmaß der Sprachwissenschaft hinweisen.

Am dritten Tag der Tagung in der ersten Sektion präsentierte Roman Opiłowski (Wrocław) das Referat unter dem Titel: Medienlinguistik 3.0. Aufgaben und Herausforderungen am Beispiel von Online-Kommunikationsformen. Im Vortrag wurde versucht, die wichtigsten kommunikativ-sozialen Tendenzen in der Online-Kommunikation darzustellen. Danach folgte die Präsentation Katarzyna Siewert-Kowalkowska (Bydgoszcz): Gesichter des Terrorismus am Beispiel der ausgewählten Internetbilder. Paweł Rybszleger (Poznań) sprach zum Thema: Selbstdarstellung auf Twitter als Bestandteil einer Online-Identität und Kommunikationshandlung. Dieser Vortrag konzentrierte sich auf die linguistische Analyse von Selbstdarstellungsmodellen auf Twitter. Dann sprach Michał Smułczyńki (Wrocław) zum Thema: Wo liegen die Grenzen der Hass-Sprache? Kommentare zu Anschlägen in London und Manchester in Sozialen Netzwerken in Deutschland Dänemark und Polen. Der nächste Vortrag von Bernd G. Bauske (Mainz) thematisierte Übersetzungen von Arno Schmidt. Danach folgte die Präsentation von Krzysztof Huszcza (Wrocław) zum Thema: Literatur grenzenlos. Zur überregionalen Entwicklung der niederösterreichischen Zeitschrift, Podium‘. Das letzte Referat in dieser Sektion wurde von Paulina Kluczna (Wrocław) zum Thema: Zum Wesen der Possessiupronomen in Quenya und Sindarin. Eine einleitende Analyse ihrer Verwendung und Bedeutung aufgrund des literarischen Schaffens von J. R. R. Tolkien. Sie unternahm den Versuch, Bildung der Possessivpronomina zu vergleichen.

Die zweite Sektion fing mit dem Vortrag von Grażyna Łopuszańska (Gdańsk) zum Thema: Linguistische Probleme in der maschinellen Übersetzung an. Danach folgte eine Präsentation von Margarete Flöter-Durr (Strasbourg): Probleme der Kommunikation in der Übersetzung: Sinn, Gebrauch und Relevanz. Dann wurde das Referat von Małgorzata Sieradzka (Rzeszów) zum Thema: Übersetzngsverfahren: Grenzen der Übersetzbarkeit oder Freiraum für den Übersetzer? gehalten. Dieser Beitrag wurde ausgewählten Problemen der Übersetzung gewidmet. Dann kam Jacek Tomasz Kuchta (Gdańsk) mit seinem Vortrag: Die Sprache als Schlüssel zur Welt. Das sprachliche Weltbild in der Sprachdidaktik Deutsch als Fremdsprache / Polnisch als Fremdsprache zu Wort. Tadeusz Zuchewicz (Zielona Góra) sprach zum Thema: Schriftliches Problemlösen im DaF-Studium: Vom Satz zum Absatz zum 
Text. Der nächste Beitrag wurde von Marcelina Kałasznik (Wrocław) zum Thema: Formen der (Un)Höflichkeit in Überschriften der deutschen und polnischen Presse. Im Mittelpunkt des Referats befanden sich Überschriften in Pressetypen. Nach diesem Vortrag sprach Anna Jeremkiewicz-Kwiatkowska (Rzeszów) über Stellungsmöglichkeiten der Fokuspartikel ,nur'(tylko, zaledwie) und ihr Verhältnis zum Bezugsausdruck - im Deutschen und Polnischen. Danach folgte die Präsentation von Artur Tworek (Wrocław): Soziophonetik - Grenzfall einer phonetischen / sprachwissenschaftlichen Subdisziplin? Er versuchte die Frage zu beantworten, wie Soziophonetik zu definieren ist, welche Forschungsfelder sie eröffnet und welche Anwendungsmöglichkeiten sie anbietet. Dann sprach Małgorzata Żytyńska (Łódź) über Phonetischen Etüden - zwar abseits der Sprachforschung doch im Dienste der Sprachdidaktik. Der letzte Beitrag in dieser Sektion wurde von Zuzanna Czerwonka (Wrocław) zum Thema: Die phonetische Grenze zwischen Sprachen überschreiten: Die Epenthese von Gleitlauten [j] und [w] als Versuch der Spannungsnachahmung in der Aussprache der Niederländischlernenden Polen gehalten.

In der dritten Sektion sprach Martine Dalmas (Paris) zum Thema: Fernglas und Mikroskop zugleich: neue Erkenntnisse durch Korpora. Danach folgte die Präsentation Jarochna Dąbrowska-Burkhardt (Zielona Góra): Das Bild der Griechen in der deutschen Presse. Stereotype und ihr sprachlicher Ausdruck im Sommer 2015. Ihre Untersuchung widmete sich konkreten sprachlichen Realisierungen von Stereotypen über Griechenland und seine Bevölkerung im öffentlichen Diskurs der Bundesrepublik Deutschland. Das nächste Referat von Monika Schönherr (Zielona Góra) war zum Thema: Zur Diachronie der afiniten Verbalperiphrasen. Danach sprach Jürgen Ehrenmüller (Pilsen) über ,Spielerische ' Grenzüberwindungen: Die Spielmetaphorik im Gegenwartsdeutschen als Beispiel für eine zentrale Strategie der Weltmodellierung und ,Spracherweiterung“. Er zeigte, wie Metaphorik als zentrale sprachliche Strategie genutzt wird. Dann kam Janusz Stopyra (Wrocław) mit seinem Beitrag zum Thema: Einige Überlegungen zu „Grenzfällen morphologischer Einheiten“ von Nanna Fuhrhop zu Wort. Der nächste Vortrag wurde von Joanna Pędzisz (Lublin) und Przemysław Staniewski (Wrocław) zum Thema: Figurativität in der Sprache der Tänzer gehalten. Dann sprach Michał Sobczak (Bydgoszcz): Sowjetdeutsche Sprache in der Zwischenkriegszeit: Forschungsstand und Forschungsperspektiven. Die nächste Präsentation wurde von Elżbieta Dziurewicz (Sosnowiec) zum Thema: Neue Wege in der Phraseologieforschung. Plädoyer für eine korpusbasierte Arbeit dargestellt. Im Referat wurde auf Potential der Korpora in der Phraseologieforschung näher eingegangen. Danach folgte ein Beitrag von Olena Byelozyorova (Charkiw): Andeutung als Euphemisierungsstrategie für verbale Tabus. Der Vortrag wurde dem Phänomenbereich der indirekten Kommunikation und zwar ihrem Sonderfall - Andeutungen gewidmet. Das letzte Referat der dritten Sektion wurde von Renata Kozieł (Sosnowiec): Leichte Sprache - Zur Charakteristik und gesellschaftlicher Bedeutung eines neuen sprachlichen Phänomens gehalten. Dieser Vortrag setzte sich zum Ziel auf die Spezifik des Phänomens der Leichten Sprache aufmerksam zu machen.

In der Sektion Nummer vier sprach Rafał Szubert (Wrocław) zum Thema: Zur textuellen Konstitution von Terminologie oder wie ein Fachwortschatz in einem Fachtext eingeführt wird. Dann kam Gabriela Nitka (Rzeszów) mit ihrer Präsentation: Semantische und syntaktisch-morphologische Eigenschaften der deutschen und polnischen Gerichtsurteile zu Wort. 
Im Mittelpunkt des Beitrags standen semantische Mechanismen, syntaktische Konstruktionen und morphologische Paradigma, die in der deutschen und polnischen Fachsprache der Gerichtsurteile vorzufinden sind. Danach folgte Renata Nadobnik (Gorzów Wielkopolski) zum Thema: Die Aussagekraft der Bilder in deutsch-polnischen Sprachführern. Der Beitrag wurde auf grafische Inhalte der deutsch-polnischen Sprachführer fokussiert. Der nächste Vortrag wurde von Marta Rogozińska (Wrocław): Zum Sprecher-HörerVerhältnis in deutschen und polnischen Konferenzvorträgen gehalten. Es wurde analysiert, auf welcher Art und Weise die Vortragenden einen Bezug zum Rezipienten herstellen. Dann sprach Adam Gołębiowski (Wrocław) zum Thema: Zu Grenzen der Attribuierung in wissenschaftlichen Texten. Danach folgte die Präsentation von Anna Gondek (Wrocław): Kreativität und Originalität bei der Benennung von kulinarischen Produkten am Beispiel der Namen für polnische Wurstprodukte. Das nächste Referat wurde von Józef Jarosz (Wrocław) zum Thema: Zu Grenzen des Kulturtransfers in zweisprachigen Wörterbüchern gehalten. Er konzentrierte sich auf zweisprachige dänische, polnische und deutsche Wörterbücher. Danach sprach Justyna Dolińska (Sosnowiec) zum Thema Zwischen Wortbildung und Syntax - Partikelverben. Dann kam Blanka Datinská (Brünn): Bundeskanzlerin und Krankenbruder? Movierung und das soziologische Grenzgebiet der deutschen Sprachwissenschaft zu Wort. Der Vortrag stellte einen Versuch dar, diese Frage anhand konkreter Untersuchung aus soziolinguistischer Sicht zu diskutieren. Der letzte Beitrag in dieser Sektion wurde von Markéta Valičková (Brünn): Möglichkeiten der korpusbasierten sprachwissenschaftlichen Analyse. Am Beispiel von Konstruktionen mit "lassen" gehalten.

Die thematische Vielfalt der Beiträge bot den Teilnehmern Gelegenheit an, an vielen interessanten Diskussionen teilzunehmen, eigene wissenschaftliche Ansätze vorzustellen und weitere Forschungsperspektiven anzubieten. Die große Resonanz, auf die das Rahmenthema der Tagung stieß, zeugte von einem großen Interesse der Forscher. An der Tagung nahmen auch Doktoranden und Studenten teil. Die Organisatoren der Tagung beabsichtigen, die Beiträge, die auf die während der Tagung gehaltenen Referate zurückgehen, in der Zeitschrift „Linguistische Treffen in Wrocław“ zu veröffentlichen.

Krystian Suchorab / 273098@uwr.edu.pl

Universität Wrocław

Instytut Filologii Germańskiej

PI. Nankiera 15b

50-140 Wrocław

Polen 